\title{
Immediate Accelerated Shoulder Rehabilitation (IASR) vs. Standard Protocol following Reverse Total Shoulder Arthroplasty: A Randomized Controlled Trial.
}

\section{Ryan Hatch \\ University of Minnesota \\ Avias Raja \\ TRIA Orthopaedic Center \\ Lauren Smudge \\ TRIA Orthopaedic Center \\ Travis Cambronne \\ TRIA Orthopaedic Center \\ Michael Walsh \\ TRIA Orthopaedic Center}

Paul DeVito ( $\sim$ Paul.DeVito@tria.com )

TRIA Orthopaedic Center https://orcid.org/0000-0002-6252-8949

\section{Study protocol}

Keywords: Rehabilitation; Reverse Shoulder Arthroplasty; Accelerated; Protected; Immobilization

Posted Date: February 11th, 2020

DOI: https://doi.org/10.21203/rs.2.23073/v1

License: (c) (i) This work is licensed under a Creative Commons Attribution 4.0 International License. Read Full License 


\section{Abstract}

Background: Recently, the reverse total shoulder arthroplasty (rTSA) became the most frequently performed form of shoulder replacement in the United States and abroad. While physical therapy is universally accepted and routinely practiced, published postoperative rehabilitation guidelines and modifications are based on protocols developed in 1975. Since then, the principle components and temporal concepts of timing and intensity of postoperative rehabilitation and protected immobilization have been controversially applied and expertly discussed without a prevailing consensus. Comparative literature that prospectively addresses treatment differences, influences on outcomes, and superiority or inferiority versus control does not exist. Thus, the objective of this pilot study is to compare an accelerated and immediate active shoulder rehabilitation (IASR) rehabilitation program with standard care in patients following rTSA.

Methods: A prospective, randomized, controlled, parallel-designed, two-armed, non-blinded, superiority pilot trial. The comparator arms are an accelerated rehabilitation (IASR) strategy versus standardized care following rTSA (CPT: 23472). The primary outcome measures will be the change from pre-surgical baseline to 52 weeks postsurgery in the American Shoulder and Elbow (ASES) score, Simple Shoulder Test (SST), and global health assessment (PROMIS-10. Secondary and tertiary outcomes will assess subscapularis integrity, implant status, and overall rehabilitation costs compared to formal control. Patients will be recruited from a single site (TRIA Orthopaedic Center, Bloomington, MN) neighboring the fourteenth largest US metropolitan area.

Discussion: This trial will be the first study to compare the efficacy and safety of two different postoperative rehabilitation strategies for patients following rTSA. Further, this will be the first study to investigate an accelerated rehabilitation program without a period of protected immobilization following rTSA. Finally, the results will assist in the shared clinical and surgical treatment decision-making process.

\section{Introduction}

\section{Background and rationale $\{6 a\}$}

Bursting onto the United States (US) healthcare scene in 2003, the reverse total shoulder arthroplasty (rTSA) has garnered widespread approval, popularity, and endorsement throughout the orthopaedic community. Although a relatively new surgical technique, the procedure surpassed the absolute number of primary anatomic total shoulder arthroplasties (aTSA) performed in the United States in 2014 (45.9\% vs. 43.6\%), United Kingdom in 2013 (35.1\% vs. 33.4\%), Australia in 2015 (50.8\% vs. 46.7\%), and Norway from 1994 to 2005 (76.3\% vs. 23.7\%). (1-4)

However, despite orthopaedic acceptance, significant disagreement exists regarding rTSA rehabilitation guidelines following surgery. (5-12) Traditionally, postoperative rehabilitation has centered on staged physiotherapy followed by early protective and restrictive joint immobilization. These principles were developed to avoid immediate postsurgical rehabilitation concerns like prosthesis instability/dislocation and acromial overload, stress reaction, and/or stress fracture commonly associated with deltoid over tension. (11,13-15) Poorly understood complications that have been previously reported to occur at rates of $0-8 \%(16-18)$ and $2.8 \%$ (19) respectively.

Despite these concerns, studies demonstrating immobilization type/length and precisely defined physiotherapy progression criteria are limited to expert opinion. As a consequence, conservative postoperative rehabilitation orthodoxy has recently been challenged by a select few. $(6,7,10,20,21)$ These outliers adopted an accelerated rehabilitation approach, shortening post-surgical immobilization to a minimum of two weeks rather than the 
"agreed-upon" expert recommendation of four to six weeks. Coupled with early initiation of range of motion (ROM) training, their protocol sought to optimize and restore shoulder mobility, eliminate postoperative joint capsule adhesions, and scarring between muscle groups; complications commonly associated with prolonged and restricted shoulder immobilization. $\{\ldots\}(22,23)$

These foreword thinking physicians recognized patients exceeding previously established human boundaries if given a chance. Nevertheless, one must question: What is truly possible? Are we creating fictional limitations? Barriers that prevent patients from pushing beyond their limits? To date, we are not aware of any literature describing immediate active shoulder rehabilitation (IASR) training without the utilization of a protective immobilizer, $(16,24,25)$ abduction pillow, (26-29) sling, $(5-7,9,10,21,30-32)$ or "simple sling" $(20,33-38)$ following rTSA. Therefore, the purpose of this randomized controlled pilot study was to evaluate and compare the IASR protocol to current institutional outpatient controls. We hypothesize that the IASR will offer an accelerated return to independence, superior post-operative patient-reported out measures (PROMs), shoulder ROM, and patient satisfaction. Further, the IASR protocol will quantitatively demonstrate a reduction in the number of formal rehabilitation visits. Equating to enhanced cost-effectiveness and overall value compared to control.

\section{Objectives $\{7\}$}

Objective 1: To compare 1-year postoperative shoulder outcome measures in the form of the American Shoulder and Elbow Score (ASES), Simple Shoulder Test (SST), global health assessment (PROMIS Global-10), and shoulder ROM between patients randomized to the IASR (Experimental group) or current institutionally accepted rehabilitation group (Control group).

Hypothesis: The IASR protocol will result in superior ASES, SST, PROMIS Global-10, and shoulder ROM scores at 1year follow-up compared to control.

Rationale: Patients in the accelerated rehabilitation protocol group will regain ROM and strength at a faster pace than those in the rehabilitation control group.

Objective 2: To determine the safety and outcome differences of the IASR protocol compared to the control by assessing the subscapularis tear rate, prosthesis dislocation, and hardware failure at 1-year follow-up with radiographic and ultrasound analysis.

Hypothesis: Patients in both groups will have similar subscapularis tear, prosthesis dislocation, and hardware failure rates. However, patients with identifiable subscapularis tears will report lower functional outcomes scores coupled with inferior ROM.

Rationale: A current relationship between postoperative subscapularis tears and rehabilitation outcome measures post rTSA is unknown.

Objective 3:To evaluate the cost-effectiveness and overall value of implementing the IASR protocol compared to control.

Hypothesis: The IASR protocol will quantitively reduce formal rehabilitation and physical therapy (PT) visits. Thus, implementation will demonstrate superior cost-savings and overall value compared to control. 
Rationale: In today's medical landscape, healthcare providers, insurance companies, and patients are continually looking for faster, safer, and cheaper healthcare and surgical alternatives.

\section{Trial design $\{8\}$}

This study protocol is a prospective, randomized, controlled, parallel-designed, two-armed, non-blinded, superiority pilot trial. The comparator arms are an accelerated rehabilitation (IASR) strategy versus standardized care following rTSA (CPT: 23472). Outcomes (Table 1) will be assessed at baseline, 2, 6, 12, 26, and 52 weeks postsurgery with the 52-week assessment serving as the principal outcome assessor. The study protocol was developed based off and adhered to the Standard Protocol Items: Recommendations for Interventional Trials (SPIRIT) guidelines. (39)

Central ethical approval was confirmed from the HealthPartners Institution Review Board (ref approval no. A18259) on 20 September 2018. Funding was provided by an Internal Grant award from the HealthPartners Institute. The trial was registered at the National Institutes of Health (NIH), United States National Library of Medicine (NLM) clinical trial database (ClinicalTrials.gov, Identifier: NCT03804853) on 15 January 2019. All participants will be asked to sign an informed consent prior to initiating the study.

\section{Methods}

\section{Participants, interventions and outcomes}

\section{Study setting \{9\}}

Patients will be recruited from a single site (TRIA Orthopaedic Center, Bloomington, MN) neighboring the fourteenth largest US metropolitan area. (40) Patients will be considered for enrollment in the study if they meet all of the inclusion criteria and none of the exclusion criteria. The criteria are based on clinical (history and physical examination), intraoperative, and postoperative findings. Patients who meet the clinical inclusion and exclusion criteria will be enrolled provisionally until the surgery has been performed. Information pertaining to the study site location can be found at: URL:https://clinicaltrials.gov/ct2/show/NCT03804853?cond = reverse+total+shoulder+arthroplasty\&draw $=1 \&$ rank $=5$

\section{Eligibility criteria $\{10\}$}

Inclusion criteria: Any patient who meets all of the following criteria may be included in this study. The patient must be:

- > 55 years of age.

- Candidate for a primary rTSA. (CPT code 23472)

- Capable of completing self-administered questionnaires.

- Be willing and able to return for all study-related follow-up procedures.

- Able and willing to give informed consent.

- Proficient in the English language. 


\section{In-Clinic, the patient cannot have/be:}

- Been treated with a primary rTSA for proximal humeral fracture.

- A surgical history including ipsilateral aTSA, rTSA, hemiarthroplasty, or instability repair.

- An active bacterial infection of the shoulder.

- Additional ipsilateral or contralateral upper limb pathology, not including the shoulder, which requires active treatment (i.e., surgery or brace).

- Taken anticoagulants within ten days prior to surgery.

- Peripheral neuropathy or other neurological disorders that may impair their ability to ambulate.

- On worker's compensation.

- Any condition requiring chemotherapy.

- Active tobacco user or former tobacco user who is not free of using tobacco for eight weeks.

- A current drug or alcohol abuser.

- A major medical illness. (life expectancy less than two years or unacceptably high operative risk)

- Suspicion of cervical radiculopathy or myelopathy.

- Deltoid insufficiency on physical examination.

\section{Intra-operative, the patient cannot have/be:}

- An iatrogenic glenoid fracture.

\section{Post-operative, the patient cannot have/be:}

- A neurological injury of the upper extremity.

- Complications from Primary rTSA (i.e., postoperative infection, bleeding, hardware failure).

If a patient is a surgical candidate for a primary rTSA, their surgeon will evaluate him/her to determine if they are eligible for inclusion in this study. If the patient fulfills the eligibility criteria, they will be referred to the principal investigator.

\section{Who will take informed consent? \{26a\}}

The principal investigator performs the final eligibility assessment, provide the patient with study specifics, and ask for consent to participate. It will be made clear that by agreeing to take part in the study, the patient will be advised to follow the postoperative physical rehabilitation protocol they were randomized to. The patient is further informed that they can refuse to participate in the study and/or wish to withdraw from the study at any time. After the patient has their questions answered and has been consented, following ample time to decide, the patient and the study staff with whom the informed consent was discussed will sign and date the informed consent form. 
The study participant will be queried on the intent of the study and follow-up time points to assess their understanding of the consent. A copy will be provided to the study patient for future reference. The patient will be considered enrolled in the study and will be randomly assigned to one of the two postoperative physical rehabilitation protocols following a randomization scheme produced by a random number generator. Neither the patient nor the physician will be blinded to the intervention. If the patient does not consent to the study, they will receive appropriate treatment as determined by the surgeon.

\section{Additional consent provisions for collection and use of participant data and biological specimens $\{26 \mathrm{~b}\}$}

Not applicable

\section{Interventions}

\section{Explanation for the choice of comparators $\{6 \mathrm{~b}\}$}

The comparator arms (Figure 1) are an accelerated rehabilitation (IASR) strategy versus standardized care following rTSA (CPT: 23472). Outcomes will be assessed at baseline, 2, 6, 12, 26, and 52 weeks post-surgery with the 52-week assessment serving as the principal outcome assessor.

\section{Intervention description \{11a\}}

All rTSA will be performed and admitted as an inpatient procedure by a non-blinded, dual-fellowship (Shoulder and Elbow, Sports Medicine) trained orthopaedic surgeon with 14 years of experience in this field. A deltopectoral approach will be employed. The biceps tendon will be tenotomized, and the circumflex vessels will be cauterized or ligated. Next, the humeral head will be dislocated and the rotator cuff will be inspected for tears. The humeral head will then be reamed up to size for the humeral broach and final component cortical contact. A cutting jig will be placed and the humeral head removed using an oscillating saw with the cut at 30 degrees retroversion at the level of the insertion of the rotator cuff. Broaching of the humeral canal will then take place to size the broach and final humeral stem implant appropriately. Exposure of the glenoid by retraction of the humerus and capsulectomy and labrectomy then will be completed. A guide pin will be inserted in the glenoid in 10 degrees of cephalic tilt and the glenoid reamed to a 3 O'clock to 9 O'clock subcortical ream. A single implant system (Comprehensive Reverse Shoulder System; Zimmer Biomet, Warsaw, Ind, USA) will be used for all study patients.

A $25 \mathrm{~mm}$ glenosphere base plate will be inserted and secured with a $6.5 \mathrm{~mm}$ center screw and 4 peripheral screws of both locking and cortical design. A glensosphere of 36 or $41 \mathrm{~mm}$ will be selected based on patient anatomy and inserted with a taper fit locking mechanism to the glenosphere base plate. The trial broach will be removed and repair stitches placed around the subscapularis osteotomy site. The final size-matched stem will be inserted in 30 degrees of retroversion followed by component trialing conducted. A tray/polyethylene combination that provides appropriate soft tissue tension and range of motion will be selected and assembled. These final components will be inserted onto the stem and the construct reduced. Subsequent osteotomy repair and biceps tenodesis to the upper pectoralis will be conducted. 
A non-blinded, inpatient PT evaluation is scheduled for all patient's post-op day 1. Randomization grouping is revealed to the patient at that time. Patients are appropriately educated on exercise execution and restrictions unique to their rehabilitation group and instructed to follow said protocol regimen. Patients are given supporting documentation specific to their rehabilitation grouping that details individual exercise descriptions and frequencies to complete on a temporal basis.

IASR Protocol (Additional file 1): The IASR is a single-phase 6-week protocol that adheres to home rehabilitation. Patients begin immediate pendulum swings, seated scapular retraction exercises, four-quadrant-stretching, seated or standing table slides, and full aROM of the shoulder, hand, wrist, and elbow post-op day 1 in the hospital. Immediate $\mathrm{pROM}$ and aROM is unlimited within pain-free ranges. Patients are instructed that sling immobilization is not necessary. However, a sling can be used as needed for pain control. Protocol endpoint goals include achieving > 120 degrees of passive flexion, > 45 degrees of passive external rotation (at side), active standing flexion to $>100$ degrees with minimal compensation, a resting pain-free state, and restoring functional use of involved extremity for all required activities of daily living including return to work when cleared by surgeon.

Standardized Protocol (Additional file 2):The standardized TRIA physical therapy protocol follows a traditional twophase approach. $(9,18)$ Patients begin therapy followed by a gradual return to aROM of the shoulder beginning at post-op week 6 , calling for 18 total physical therapy visits over 12 weeks. Therapy will transition from two sessions per week in Phase I (0-6 weeks) to one session per week in Phase II (6-12 weeks).

- Phase 1 (0-6 weeks): Patients are instructed to wear a sling at all times for four weeks, except for dressing changes, hygiene, and formal therapy. Motion restrictions and precautions include aROM, internal rotation, internal isometric rotation, internal rotation progressive resistive exercises (PREs), shoulder extension with adduction and internal rotation, and shoulder hyperextension. Patients, however, are permitted to begin pROM as tolerated prohibiting internal rotation in the scapular plane, aROM of the elbow wrist and hand, pendulum swings, submaximal scapular exercises (retraction, depression, circles) and submaximal pain-free deltoid isometrics.

- Phase 1 Goals: To maximize surgical repair protection, maintain mobility of the surrounding joints, and control swelling and pain.

- Phase 2 (6-12 weeks): Patients begin a progressive sit to stand active assistive ROM (aaROM) and aROM in all planes, and progressive rhythmic scapular and glenohumeral stabilization exercises. Post-op week 10 introduces isometric to isotonic progression of external and internal rotation, and PREs in all planes within ROM restrictions; emphasizing the deltoid.

- Phase 2 Goals: To achieve 80-120 degrees of elevation, 30 degrees of external rotation, and to restore the functional use of the involved extremity for all required activities of daily living, including return to work when cleared by the surgeon.

\section{Criteria for discontinuing or modifying allocated interventions $\{11 b\}$}

Deviations will be documented using our standardized protocol deviation form, and the protocol deviation log will be updated. The protocol deviation form is signed by the coordinator documenting the deviation and the PI or research manager. At that time, a determination of subject status will be made-continue to follow, use data already 
collected, withdraw, lost to follow-up. Finally, the study may be stopped early by the investigator or the FDA for any of the following reasons:

- For the patients' safety

- If the patient does not follow the instructions for the study

- Hardware loosening

\section{Strategies to improve adherence to interventions $\{11 \mathrm{c}\}$}

The TRIA research team will facilitate protocol intervention adherence. Through a multifaceted collaborative effort, the research team developed the protocol, study delivery system, operating procedures, training, survey database, and patient report forms. Further, to ensure home therapy compliance, patients will receive a daily text message alert from a HealthPartners text reminding service.

\section{Relevant concomitant care permitted or prohibited during the trial $\{11 d\}$}

Physical therapy may occur in off-site clinics and centers. However, we would prefer if patients received their physical therapy at a TRIA/HealthPartners location conveniently located close to the patients' residence.

\section{Provisions for post-trial care $\{30\}$}

If a patient gets hurt and/or sick from being in this study, they are instructed to seek medical treatment and inform the investigator as soon as possible. TRIA will bill the patient's insurance, if applicable, and the patient will be responsible for their usual copay or deductible. If the patient has Medicare, TRIA will facilitate the delivery of patient information that identifies said patient to the Medicare system. If a patient does not have insurance or if their insurance does not cover their treatment, TRIA will bill the patient for the costs of the treatment.

\section{Outcomes $\{12\}$}

- American Shoulder and Elbow Score (ASES): The ASES is a standardized form used for the assessment and evaluation of patient shoulder function. Patients evaluate pain, instability, and their activities of daily living. Physicians record demographic details, ROM measurements, specific physical signs, stability, and strength scores. The ASES score validity, reliability, and responsiveness has been assessed in a variety of shoulder problems including rotator cuff disease, glenohumeral arthritis, shoulder instability, and shoulder arthroplasty. $(41,42)$ The assessment consists of 11 items, one item for pain and 10 items for function. The score for each subscale is out of $50(0-50)$, with a higher score indicating a better outcome. The total score is a sum of both subscales, ranging between $0-100$, using the following formula, Total score $=((10-$ VAS pain $) \times 5)+(5 / 3 \times$ sum of function items).

Simple Shoulder Test (SST): The SST is a series of 12 "yes" or "no" questions the patient answers about the function of the involved shoulder. The answers to these questions provide a standardized way of recording the function of a shoulder before and after treatment. (43) There are 2 items on function related to pain, 7 items about 
functions/strength and 3 items about range of motion. There are no subscales. A "yes" response is 1 point and a "no" response is 0 point. The following formula is used to calculate a score out of $100(0-100)$, total = number of "yes" items/number of completed items $\times 100=\%$ "yes" responses. A higher score indicates better function from a scale of $0-100$.

- PROMIS Global-10: The PROMIS Global-10 is a publicly available global health assessment tool that allows measurements of symptoms, functioning, and healthcare-related quality of life (HRQoL) for a wide variety of chronic diseases and conditions. The PROMIS Global-10 short form consists of 10 items that assess general domains of health and functioning, including overall physical health, mental health, social health, pain, fatigue, and overall perceived quality of life. 7 questions inquire about health in "general," and 3 questions assess emotional problems, fatigue and pain in the last 7 days. Raw subscale scores for physical and mental health each are calculated out of 20 . The raw score are converted to T scores for physical and mental health using the following formulas: the Global Physical Health score is generated by summing responses to Global03, Global06, Global07rescored, and Global08rescored. The Global Mental Health score is generated by summing responses to Global02, Global04, Global05, and Global10rescored. T scores range from 0-100. A high score represents a better outcome. There is not a total score for this tool. The 10 questions of the Global-10 have primarily been adapted from other frequently used "legacy" measures such as the SF-36 and EQ-5D. (44)

- Range of Motion (ROM): of the shoulder is an objective measure of shoulder function. A blinded examiner will measure the range of motion in forward elevation, external rotation in the neutral position external rotation with the arm in abduction, internal rotation in the neutral position, and internal rotation with the arm in abduction. A goniometer will be used measuring to the nearest degree. The range of motion for forward elevation will range between 0-180 degrees with a higher number indicating a better outcome. External and internal rotation ranges from 0-90 degrees with a higher number indicating a better outcome.

Radiographic (X-Ray) Measures: A musculoskeletal radiologist and dual-fellowship trained orthopaedic surgeon will evaluate anteroposterior, axillary, and scapular-y view radiographs. The prosthesis review will adopt the rTSA radiographic assessment checklist published by Roberts et al. (45) Scapular notching will be evaluated according to the Sirveaux classification. (46) Humeral radiolucent lines will be classified according to the Sperling System. $(47,48)$ The inferior overhang of the glenosphere will be measured according to Bigorre et al. $(49)$ And implant loosening will adhere to the principles published by Melis et al. (50)

Shoulder Ultrasound: All ultrasonograms will be obtained in real-time with use of an ATL HDI 3000 scanner (Advanced Technologies Laboratories, Bothell, WA) or a Siemens Elegra scanner (Siemens Medical Systems, Issaquah, WA), and a variable high-frequency linear-array transducer (7.5 to ten megahertz) to assess subscapularis integrity. All patients will have standardized bilateral ultrasonography of the shoulder, performed by one of two radiologists who experienced with the technique. $(51,52)$

- A finding of a full-thickness rotator cuff tear will be recorded when the rotator cuff cannot be visualized due to either a complete cuff avulsion and retraction under the acromion or a focal defect in the cuff created by a variable degree of retraction of the torn tendon ends. If a rotator cuff tear is not visualized, the deltoid muscle will be compressed against the cuff with the transducer to separate the torn tendon ends at the site of a nonretracted tear. A finding of a partial-thickness tear is present if minimal flattening of the bursal side of the rotator cuff (a bursal-side partial-thickness tear) or a distinct hypoechoic or mixed hyperechoic and hypoechoic defect visualized in both the longitudinal and the transverse plane at the deep articular side of the rotator cuff 
(an articular-side partial-thickness tear). The extent of the rotator cuff tear will be determined with transverse measurements. Both radiologists will report the ultrasound, regardless of who performs it.

\section{Cost of Care Measures:}

- Number of PT visits.

- Cost of PT visits to the patient calculated as mentioned below:

- Request a list of all patients that had an rTSA and rehab at TRIA. At a per-patient level, we will see the number of visits and cost per PT session. Then take the total cost/total number of sessions to get an average. Total Cost of PT = Average cost per session $x$ Number of PT visits 
Table 1 Outcome data collection schedule

\begin{tabular}{|c|c|c|c|c|c|c|c|}
\hline & Baseline & Op day & $\begin{array}{l}\text { Post-op } 2 \\
\text { weeks }\end{array}$ & $\begin{array}{l}\text { Post-op } 6 \\
\text { weeks }\end{array}$ & $\begin{array}{l}\text { Post-op } 3 \\
\text { months }\end{array}$ & $\begin{array}{l}\text { Post-op } 6 \\
\text { months }\end{array}$ & $\begin{array}{l}\text { Post-op } 1 \\
\text { year }\end{array}$ \\
\hline \multicolumn{8}{|l|}{$\begin{array}{l}\text { Visit } \\
\text { Description }\end{array}$} \\
\hline $\begin{array}{l}\text { Healthcare } \\
\text { Provider }\end{array}$ & OS & OS & OS & OS & OS & OS & OS \\
\hline $\begin{array}{l}\text { Objective of } \\
\text { visit }\end{array}$ & $\begin{array}{l}\text { Potential } \\
\text { study } \\
\text { patient for } \\
\text { study } \\
\text { enrollment. } \\
\text { Get } \\
\text { informed } \\
\text { consent. } \\
\text { Collect } \\
\text { PROM }\end{array}$ & & $\begin{array}{l}\text { Collect } \\
\text { PROM, } \\
\text { financial } \\
\text { data. } \\
\text { Order XR }\end{array}$ & $\begin{array}{l}\text { Collect } \\
\text { PROM, } \\
\text { financial } \\
\text { data. } \\
\text { Order XR }\end{array}$ & $\begin{array}{l}\text { Collect } \\
\text { PROM, } \\
\text { financial } \\
\text { data. } \\
\text { Order XR }\end{array}$ & $\begin{array}{l}\text { Collect } \\
\text { PROM, } \\
\text { financial } \\
\text { data. } \\
\text { Order XR }\end{array}$ & $\begin{array}{l}\text { Collect } \\
\text { PROM, } \\
\text { financial } \\
\text { data. } \\
\text { Order XR }\end{array}$ \\
\hline Visit Length & $30 \min$ & & $30-45$ min & $30-45$ min & $30-45$ min & $30-45$ min & 60-90 min \\
\hline Coverage & $\begin{array}{l}\text { Patient } \\
\text { Insurance }\end{array}$ & \begin{tabular}{|l|} 
Patient \\
Insurance \\
\end{tabular} & $\begin{array}{l}\text { Patient } \\
\text { Insurance }\end{array}$ & $\begin{array}{l}\text { Patient } \\
\text { Insurance }\end{array}$ & $\begin{array}{l}\text { Patient } \\
\text { Insurance }\end{array}$ & $\begin{array}{l}\text { Patient } \\
\text { Insurance }\end{array}$ & $\begin{array}{l}\text { Patient } \\
\text { Insurance }\end{array}$ \\
\hline \multicolumn{8}{|l|}{ PROM } \\
\hline ASES & $\mathrm{X}$ & & $\mathrm{X}$ & $\mathrm{X}$ & $\mathrm{X}$ & $\mathrm{X}$ & $\mathrm{X}$ \\
\hline$\overline{\text { SST }}$ & $\bar{X}$ & & $\mathrm{X}$ & $\mathrm{X}$ & $\mathrm{X}$ & $\mathrm{X}$ & $\mathrm{X}$ \\
\hline PROMIS-10 & $\mathrm{X}$ & & $\mathrm{X}$ & $\mathrm{X}$ & $\mathrm{X}$ & $\mathrm{X}$ & $\mathrm{X}$ \\
\hline $\begin{array}{l}\text { ROM } \\
\text { Shoulder }\end{array}$ & $\bar{X}$ & & & & $\mathrm{X}$ & $\mathrm{X}$ & $\mathrm{X}$ \\
\hline Coverage & $\begin{array}{c}\text { Grant/ } \\
\text { Research }\end{array}$ & & $\begin{array}{c}\text { Grant/ } \\
\text { Research }\end{array}$ & $\begin{array}{c}\text { Grant/ } \\
\text { Research }\end{array}$ & $\begin{array}{c}\text { Grant/ } \\
\text { Research }\end{array}$ & $\begin{array}{c}\text { Grant/ } \\
\text { Research }\end{array}$ & $\begin{array}{c}\text { Grant/ } \\
\text { Research }\end{array}$ \\
\hline \multicolumn{8}{|l|}{$\begin{array}{l}\text { Radiological } \\
\text { X-Rays }\end{array}$} \\
\hline $\begin{array}{l}\text { Prosthesis } \\
\text { dislocation, } \\
\text { etc }\end{array}$ & & & $\mathrm{X}$ & $\mathrm{X}$ & $\mathrm{X}$ & $\mathrm{X}$ & $\mathrm{X}$ \\
\hline Coverage & & & $\begin{array}{c}\text { Patient } \\
\text { Insurance }\end{array}$ & $\begin{array}{c}\text { Patient } \\
\text { Insurance }\end{array}$ & $\begin{array}{c}\text { Grant/ } \\
\text { Research }\end{array}$ & $\begin{array}{c}\text { Patient } \\
\text { Insurance }\end{array}$ & $\begin{array}{c}\text { Grant/ } \\
\text { Research }\end{array}$ \\
\hline \multicolumn{8}{|l|}{ Imaging } \\
\hline $\begin{array}{l}\text { Hand Held } \\
\text { Ultrasound }\end{array}$ & & & & & & & $\mathrm{X}$ \\
\hline Coverage & & & & & & & $\begin{array}{c}\text { Grant/ } \\
\text { Research }\end{array}$ \\
\hline \multicolumn{8}{|l|}{ Financial } \\
\hline $\begin{array}{l}\text { Number of } \\
\text { PT visits }\end{array}$ & & & $\mathrm{X}$ & $\mathrm{X}$ & $\mathrm{X}$ & $\mathrm{X}$ & \\
\hline
\end{tabular}

Legend: OS - Orthopaedic Surgeon; PROM - Patient Reported Outcome Measure; ASES American Shoulder and Elbow Score; SST - Simple Shoulder Test; XR - X-ray Radiographs

\section{Participant timeline $\{13\}$}


Table 2 shows the expected flow of participants through recruitment, assessment, intervention, and follow-up. The patient will have to attend a total of six visits. In all these visits the patient will be asked to complete questionnaires, undergo a shoulder examination, and x-rays. An additional shoulder ultrasound will be conducted at the last visit.

The study is expected to take up to 30 months, consisting of 20 months for recruitment and randomization, 8 months for final participant follow-up, and 2 months for analysis and manuscript writing.

Table 2 Participant Timeline

\begin{tabular}{|c|c|c|c|c|c|c|}
\hline & Visit 1 & Visit 2 & $\begin{array}{l}\text { Visit } 3 \\
\end{array}$ & Visit 4 & Visit 5 & Visit 6 \\
\hline & Baseline & 2 Weeks & 6 Weeks & $\begin{array}{c}3 \\
\text { months }\end{array}$ & $\begin{array}{c}6 \\
\text { Months }\end{array}$ & 1 year \\
\hline How long is the visit? & 1 hour & 1 hour & 2 hours & 2 hours & 2 hours & $\begin{array}{c}2.5 \\
\text { hours }\end{array}$ \\
\hline Consent & $\mathrm{X}$ & & & & & \\
\hline $\begin{array}{c}\text { Demographic/ } \\
\text { Medical History }\end{array}$ & $\mathrm{X}$ & & & & & \\
\hline Height and Weight & $\bar{X}$ & & & & & \\
\hline Imaging & $\begin{array}{l}\text { x-ray and/or } \\
\text { CT scan }\end{array}$ & $\begin{array}{l}\text { x-ray } \\
\text { series }\end{array}$ & $\begin{array}{l}\text { x-ray } \\
\text { series }\end{array}$ & $\begin{array}{l}\text { x-ray } \\
\text { series }\end{array}$ & $\begin{array}{l}\text { x-ray } \\
\text { series }\end{array}$ & $\begin{array}{l}\text { x-ray } \\
\text { series }\end{array}$ \\
\hline Shoulder Exam & $\mathrm{X}$ & & & $\mathrm{X}$ & $\mathrm{X}$ & $\mathrm{X}$ \\
\hline Outcome Questionnaires & $\mathrm{X}$ & $\bar{X}$ & $\mathrm{X}$ & $\mathrm{X}$ & $\mathrm{X}$ & $\mathrm{X}$ \\
\hline Wound Check & & $\mathrm{X}$ & & & & \\
\hline Ultrasound & & $\mathrm{X}$ & $\mathrm{X}$ & $\mathrm{X}$ & $\mathrm{X}$ & $\mathrm{X}$ \\
\hline $\begin{array}{c}\text { Review Adverse } \\
\text { Events/Complications }\end{array}$ & & $\mathrm{X}$ & $\mathrm{X}$ & $\mathrm{X}$ & $\mathrm{X}$ & $\mathrm{X}$ \\
\hline $\begin{array}{c}\text { Billed to Your Insurance } \\
\text { (Standard of Care) }\end{array}$ & \multicolumn{2}{|l|}{$\mathrm{X}$} & \multicolumn{2}{|c|}{ Paid for by TRIA } & \multicolumn{2}{|c|}{$\mathrm{X}$} \\
\hline
\end{tabular}

\section{Sample Size $\{14\}$}

The calculation was based on postoperative ASES scores in a previously published comparative study. (53) To detect a clinically ASES score relevant difference of 15.8 between groups, with a mean improvement standard deviation (SD) of 20.6 , alpha of $5 \%$ and power of $80 \%, 31$ patients were needed in each group. Assuming a drop-out rate of $20 \%$, at least 37 patients were required per group. This leaves us with a total sample size of 74 . If we consider the minimal clinical important difference (MCID) of 11.4 for SST, we would need 34 patients per group to possibly reach statistical significance. (54) A total of 34 patients will be necessary to detect a $10^{\circ}$ difference in ROM of the shoulder in all planes based on an SD of $10^{\circ}$. (55) 


\section{Recruitment $\{15\}$}

TRIA Orthopaedic Center is the ideal location for patient recruitment as Dr. Walsh performs approximately 100 anatomical and reverse total shoulder arthroplasty procedures (75\%).

The research branch of the TRIA Institute is comprised of a Medical Director, Research Manager, 2 full-time Research Coordinators, a Research Assistant, a Research Fellow, a Ph.D. Research Scientist and an MD Research Scientist. The Research Coordinators and Assistant are primarily involved in the day to day research activities. All can take on the work of an additional study.

All staff listed on the study and participating in the research will attend a study kick-off meeting where the details of the study will be discussed. A review of the protocol, consent form, delegation of duties, case report forms and study procedures will be reviewed. All staff on the study have been trained by research staff and investigators on proper consent procedures and have completed CITI training.

\section{Assignment of intervention: allocation}

\section{Sequence generation $\{16 a\}$}

1:1 for treatment to control. Randomization will be conducted during the initial evaluation after consent has been confirmed via block randomization and delivered through computerized slips. A random number generator will produce the randomization assignment and scheme.

\section{Concealment mechanism \{16b\}}

A senior non-blinded, research manager will be responsible for preparing the randomization scheme and inform the operating surgeon prior to each patient's designated surgery day.

\section{Implementation $\{16 c\}$}

If a patient is a surgical candidate for a primary rTSA, their surgeon will evaluate him/her to determine if they are eligible for inclusion in this study. If the patient fulfills the eligibility criteria, they will be referred to the principal investigator. The principal investigator performs the final eligibility assessment, provide the patient with study specifics, and ask for consent to participate.

A senior non-blinded, research manager will be responsible for preparing the randomization scheme and inform the operating surgeon prior to each patient's designated surgery day. All assessors and study personnel other than the senior research manager and operating surgeon are blinded to group allocation. Patients are instructed not to communicate their group allocation to the study staff or other participating patients. All data assessed will be processed and analyzed by an institutional statistician blinded to treatment group allocation.

\section{Assignment of interventions: Blinding}




\section{Who will be blinded $\{17 \mathrm{a}\}$}

All assessors and study personnel other than the senior research manager and operating surgeon are blinded to group allocation. Patients are instructed not to communicate their group allocation to the study staff or other participating patients. All data assessed will be processed and analyzed by an institutional statistician blinded to treatment group allocation.

\section{Procedure for unblinding if needed $\{17 \mathrm{~b}\}$}

Not applicable

\section{Data collection and management}

\section{Plans for assessment and collection of outcomes $\{18 \mathrm{a}\}$}

Data collected from the follow-up will be answers to the functional shoulder (ASES, SST, and ROM), quality of life questionnaires (PROMIS Global-10), and imaging impressions at the time of consent (baseline), 2, 6, 12, 26, and 52 weeks post-surgery. If the patient misses the follow-up time points, we will consider data from 2 weeks \pm 2 days as 2 -week data, 6 weeks \pm 1 week to be 6 -week data, 12 weeks \pm 2 weeks to be 12 -week data, 26 weeks \pm 3 weeks to be 26 -week data, and 52 week \pm 4 weeks to be 52 -week data.

\section{Plans to promote participant retention and complete follow-up $\{18 b\}$}

If the patient misses one of the follow-up visits or does not come in during the timeframes specified above, we will continue to collect data from the rest of the time points unless the patient no longer wishes to participate or the principal investigator terminates their study participation.

\section{Data management $\{19\}$}

The research coordinator, or delegate, will complete a chart audit form for all patients and enter all patient data into an electronic database that only the study personnel have access to. Each study participant will be identified by their assigned study subject number in an electronic database. The research coordinator will keep the passwordprotected cross-walk files linking the participant names and medical record numbers to their subject number. Each study subject will have their own binder that includes all case report forms and their signed consent form. Patient binders and any other paper forms related to the study will be stored in a locked file cabinet in the research department at TRIA.

\section{Confidentiality $\{27\}$}


This study is being conducted in accordance with the ethical principles that are consistent with the International Conference of Harmonisation (ICH) and good clinical practice (GCP) guidelines. All study staff are fully trained on the elements of patient privacy, HIPAA, and confidentiality. All data points collected will be reviewed to ensure values are within an acceptable range. Subjects will be selected at random to ensure case report forms match electronic databases. Data will be maintained per organizational policy.

No copies of consent forms will be placed in the subject's medical, employment, or educational records. The subject will be associated with the research study in the medical record, and a flag added to their account to alert other medical staff as to their participation in the study.

Paper-based records will be kept in a secure location and only be accessible to personnel involved in the study. Computer-based files will only be made available to personnel involved in the study through the use of access privileges, passwords, and encryption. Prior to access to any study-related information, personnel will be required to sign statements agreeing to protect the security and confidentiality of identifiable information. Whenever feasible, identifiers will be removed from study-related information. Precautions are in place to ensure electronic data is secure by using passwords and encryption. A number will code data that only the investigators or research staff will have access to. All staff will be trained in the collection of data, and training will be documented and stored in the site regulatory binder.

Monitoring will be conducted to assure the rights and safety of participants, confirm the study conduct follows the guidelines of good clinical practice, assure maintenance of required documents, verify adherence to the protocol, monitor the quality of data collected, and assure accurate reporting and documentation of all adverse events. Monitoring will ensure all forms are completed. Internal monitoring will be completed on all investigator-initiated studies on a routine basis as determined departmental leadership. Findings will be regularly reported in summary format to the investigator and study team.

The senior research manager and PI or Institute Medical Director will monitor safety for this study. The research manager will routinely monitor protocol deviations. The PI or Institute Medical Director will provide oversight and review of any adverse events.

No data will be shared with external entities. Identifiers will be removed prior to statistical analysis and will be permanently deleted from the TRIA network upon publication of the results. If there is a violation of patient privacy, measures will be taken to inform the patient and to decrease the risk of further loss of privacy.

\section{Plans for collection, laboratory evaluation and storage of biological specimens for genetic or molecular analysis in this trial/future use $\{33\}$}

Not applicable

\section{Statistical methods}

\section{Statistical methods for primary and secondary outcomes $\{20 \mathrm{a}\}$}


Primary Outcome: The responder rate of the IASR group is compared to the responder rate of the control group. A patient is considered a responder if they achieve a clinically significant improvement of at least $13.6 \pm 2.2$ points in the ASES, $1.5 \pm 0.3$ points in the SST scores at postoperative 1 -year compared to baseline. $(55,56)$

Supporting Efficacy Endpoints: In addition to the primary outcome, the responder status to the PROMIS Global-10 will be calculated.

Secondary Endpoints:A comparison between the IASR and control rehabilitation groups will be made using a twosided independent students t-test with an alpha $=0.05$

- Rate of a subscapularis tear.

- Rate of prosthesis dislocation.

- Rate of Hardware failure.

- Rate of scapular notching.

- The number of total PT visits.

\section{Interim analyses $\{21 \mathrm{~b}\}$}

Two interim analyses will be performed, one at 26 and 52 weeks, so we use the Bonferroni correction to adjust the significance level for these additional tests will be utilized.

\section{Methods for additional analyses (e.g., subgroup analyses) \{20b\}}

A descriptive statistical analysis will include all counts percentages and confidence intervals for group differences in percentages. Covariate analysis of the SST score and PROMIS Global-10 will be conducted using age, gender, $\mathrm{BMI}$, tobacco use, rate of subscapularis tear, prosthesis dislocation, hardware failure, scapular notching, and the number of total PT visits.

Descriptive summaries of the patient and operative information will be generated. Means and SD will be reported for normally distributed continuous variables, medians, and ranges for non-normally distributed continuous variables; and frequencies and percentages for categorical variables. Age, symptom duration (in months), number of previous surgeries, number of comorbid conditions, BMI, and surgery duration using a 2-sample t-test or the Mann-Whitney-Wilcoxon test if normality assumptions are not met. These patients will also be compared on gender, race/ethnicity, employment, worker's compensation status, dominant hand, and smoking status using a chisquare test. Similarly, patients who drop out of the study (i.e., have baseline information only) will be compared to patients who do not drop out on the same variables using the same tests as those stated above. A generalized chisquare test will be used to evaluate differences in the number of patients responding in each category to each preoperative expectation.

- To address the primary objective, changes in scores on the quality of life measure from baseline to follow-up will be compared between the two groups using a 2-sample t-test. If the change scores are not normally distributed, we will use the Mann-Whitney-Wilcoxon test instead.

- To address the primary objective, the ASES score, Simple Shoulder test score, and PROMIS Global-10 scores, changes in scores from baseline to follow-up will be compared between the two groups using a 2-sample t- 
test. If the change scores are not normally distributed, we will use the Mann-Whitney-Wilcoxon test instead.

- To address the incidence of retear, prosthesis dislocation, and hardware failure (secondary objective 2), we will use a chi-square test to compare the number of patients in each group that had a retear confirmed by ultrasound at 12 months. To gain more information, logistic regression will be employed with retear (yes/no) as the response and any demographic or clinical variables that differ significantly between groups as predictors.

- To address the tertiary objective, changes in the number of PT visits and total cost of PT (number of PT visits $x$ average cost of a PT visit) from baseline to follow-up will be compared between the two groups using a 2sample t-test. If the change scores are not normally distributed, we will use the Mann-Whitney-Wilcoxon test instead.

Statistical analyses will be conducted with IBM-SPSS 22.0 software (IBM-SPSS, Chicago, IL). In all tests, a statistical significance of $p<0.05$ will be considered. The Kolmogorov-Smirnov test will determine normality. For comparison between groups, the unpaired Student t-test or nonparametric Mann-Whitney U test will be used for continuous variables, and the x2 test, Fisher exact test, or Mantel-Haenszel test for categorical variables. For comparison between preoperative and postoperative data and different postoperative periods within a group, the paired t-test or Wilcoxon signed-rank test will be used.

All tests will be two-sided, and a significance level of $a=0.0167$ will be used throughout. Two interim analyses will be performed, one at 26 and 52 weeks, so we use the Bonferroni correction to adjust the significance level for these additional tests will be utilized. That is, $a=0.05 / 3=0.0167$.)

\section{Methods in analysis to handle protocol non-adherence and any statistical method to handle missing data $\{20 \mathrm{c}\}$}

Monitoring will be conducted to assure the quality of data collected, and assure accurate reporting and documentation of all adverse events. Monitoring will ensure all forms are completed and data entry is valid.Missing data will be assessed and determination of the subject status will be made-continue to follow, use data already collected, withdraw, or designate lost to follow-up.

\section{Plans to give access to the full protocol, participant level-data and statistical code $\{31 \mathrm{c}\}$}

No data will be shared with external entities. Identifiers will be removed prior to statistical analysis and will be permanently deleted from the TRIA network upon publication of the results. If there is a violation of patient privacy, measures will be taken to inform the patient and to decrease the risk of further loss of privacy.

\section{Oversight and monitoring}

\section{Composition of the coordinating center and trial steering committee $\{5 d\}$}


The research branch of the TRIA Institute is comprised of a Medical Director, Research Manager, 2 full-time Research Coordinators, a Research Assistant, a Research Fellow, a Ph.D. Research Scientist and an MD Research Scientist. The Research Coordinators and Assistant are primarily involved in the day to day research activities. All have the capacity to take on the work of an additional study.

All staff listed on the study and participating in the research will attend a study kick-off meeting where the details of the study will be discussed. A review of the protocol, consent form, delegation of duties, case report forms and study procedures will be reviewed. All staff on the study have been trained by research staff and investigators on proper consent procedures and have completed CITI training.

The study proposal was approved at TRIA's monthly research meeting and reviewed by the scientific review committee. The feedback from the scientific review committee was used to make changes to the protocol to ensure appropriateness in the research methodology and delivery.

This study proposal meets our organizational goals in improving healthcare and focusing on rehabilitation from a joint replacement procedure to bring value to the individual patient and decrease cost, simultaneously.

\section{Composition of the data monitoring committee, its role and reporting structure $\{21$ a $\}$}

The investigators of this study will abide by the study protocol, all applicable laws and federal regulations, and criteria set by the reviewing IRB. A study kick-off meeting will be held prior to the commencement of the study to orient the investigators and study staff. The Data Safety Monitoring Board (DSMB) will evaluate the safety of the study. The DSMB may consider stopping the study if there is evidence of study related death, upper limb neurovascular injury, high rate of secondary surgery, deep vein thrombosis with a pulmonary embolism.

\section{The DSMB for this study is the TRIA Institute Board of directors and is responsible for:}

- Review of the research protocol informed consent documents and plans for data safety and monitoring;

- Evaluate the progress of the trial;

- Protect the safety of the study participants (review of adverse events);

- Report on the safety and progress of the trial;

- Make recommendations to the sponsor, PI, and, if required, to the Food and Drug Administration (FDA) and the IRB concerning continuation, termination or other modifications of the trial based on the observed beneficial or adverse effects of the treatment under study; and

- Ensure the confidentiality of the trial data and the results of monitoring.

- Any findings (such as protocol violations, significant safety concerns) will be promptly submitted to the IRB (and sponsor).

\section{Adverse event reporting and harms $\{22\}$}


An adverse event is any untoward medical occurrence in a study patient administered a study treatment, regardless of whether a causal relationship exists between the medical occurrence and the study treatment. An adverse event, therefore, is any unfavorable and unintended sign, symptom, abnormal assessment, or disease temporally associated with the use of study treatment, whether or not considered related to the study treatment.

Adverse events will be collected and recorded on the appropriate case report form until the patient participation is complete at the 1-year postoperative time point. Adverse events may be noted subjectively from the patient or objectively during the follow-up visits. Adverse events will be categorized (Table 3) based on the severity (mild, moderate, or severe), serious adverse events, or unexpected adverse events.

Table 3 Adverse Event Categorization

\begin{tabular}{|c|c|}
\hline $\begin{array}{c}\text { Adverse } \\
\text { Event }\end{array}$ & Definition \\
\hline Mild & Sign/symptom easily tolerated by the patient \\
\hline Moderate & Signs/symptoms that interfere with usual activities. \\
\hline Severe & Signs/symptoms that make the patient disabled from work or routine activities. \\
\hline Serious & $\begin{array}{r}\text { Signs/symptoms that is life-threatening requires hospital admission, prolonging } \\
\text { existing hospitalization, or leads to death. }\end{array}$ \\
\hline Unexpected & That is not defined under mild, moderate, severe, and serious adverse events. \\
\hline
\end{tabular}

\section{Risk Analysis}

Risks of this study focus on the period after the surgery, since study intervention will occur postoperatively. The standard of care will be provided from the initial recruitment visit until the patient is discharged from the surgery.

\section{The following risks are anticipated from the study generally:}

- Radiation exposure for all radiographic films.

\section{The following risks are anticipated from early shoulder activity and motion post rTSA:}

- Loosening of hardware.

- Peri-prosthetic bone fracture.

- Hematoma formation.

- Wound dehiscence.

- Prosthesis dislocation. 
The questionnaires used in the study may make the participants uncomfortable or upset. Participants will have the option not to answer any questions that make them uncomfortable and are free to stop a survey at any time. Investigators have chosen validated outcome tools for use in the study.

X-rays involved increase exposure to radiation. The total radiation in a knee x-ray is $0.01 \mathrm{mSv}$ (Milli Sieverts). The average person is exposed to about $3.0 \mathrm{mSv}$ of radiation per year from natural sources (naturally occurring radioactive materials and outer space). The radiation from a knee x-ray is equal to about 1.5 extra days of average exposure.

Physical performance testing: There are minimal risks associated with physical performance testing. Possible risks may include muscle fatigue, muscle or joint soreness, and in rare instances, muscle strain. To minimize physical risks, testing will be conducted under the direct supervision of a trained medical professional.

To minimize and protect the study subjects from anticipated risks mentioned, they will be counseled and instructed to contact the research study staff if they experience the above mentioned potential risk.

\section{Frequency and plans for auditing trial conduct $\{23\}$}

The research coordinator, or delegate, will complete the chart audit form for all patients and enter all the data into an electronic database that only the study personnel have access to. In the database, each study participant will be identified by their assigned study ID number. The research coordinator will keep the password-protected cross-walk file linking the participant names and MRNs to their study ID numbers. Each study subject will have their own binder that includes all case report forms and their signed consent form. These patient binders and any other paper forms related to the study will be stored in a locked file cabinet in the research department at TRIA.

\section{Plans for communicating important protocol amendments to relevant parties (e.g., trial participants, ethical committees) $\{25\}$}

Protocol amendments associated with trial safety, scientific aspects of the study, and any emerging evidence as it relates to the trial will be directly communicated to the HealthPartners IRB, DSMB, TRIA research team, and trial participants.

\section{Dissemination plans $\{31 \mathrm{a}\}$}

Irrespective of the IASR trial outcome, the results will be submitted for publication in an appropriate journal. An abstract will be developed and submitted to appropriate shoulder conferences by the principal investigator. Subsequently, the principal investigator will be responsible for manuscript composition, which the co-investigators will review and approve prior to submission. The principal investigator must approve subsequent studies utilizing data obtained from the IASR trial. The results of our study will be incorporated into our practice and replace traditional physical therapy protocols.

\section{Discussion}


Reverse shoulder arthroplasty is an established and reliable surgical procedure that relieves pain and function. However, despite the procedural success, disagreement and controversy exist regarding the principle components and concepts of postoperative rTSA rehabilitation. While adequate postoperative protection and motion restriction during rehabilitation following aTSA was found essential, (57) some believe extrapolating the same rehab guidelines post rTSA may not be proper. (58-60) Nevertheless, publications exist. Unfortunately, published literature and evidence to date is limited to expert opinions (6-11) lacking comparative analysis. This is further illustrated by a recent commission on rehabilitation post rTSA by the German Society of Shoulder and Elbow Surgery (Deutsche Gesellschaft für Schulter-und Ellenbogenchirurgie e. V., DVSE) reporting they were unable to find a single guideline, systematic review, or level 1/2 study in their literature search dating back to 1989. (61) Balanced against these dissenting views is the universal orthopaedists' acceptance therapy plays in optimizing patient outcomes, and the absolute need for high-quality prospective research. $(5,6,10,11,55)$

The IASR trial protocol will be the first randomized trial to compare the effectiveness of an accelerated shoulder rehabilitation program following rTSA compared to a traditional rehabilitation protocol adopted by our institution. $(9,18)$ It will address the lack of randomized trials assessing post-surgical and functional outcomes by providing objective, scientifically based results, and information. Information that will be essential in determining the best practice that optimizes patient functional outcomes, satisfaction, and cost-effectiveness post rTSA.

The IASR protocol study is strengthened by utilizing a single surgeon with 14 years of experience performing a high volume of primary rTSA cases annually. Additionally, the surgeon, who also serves as our primary investigator (PI) has completed research studies, including trials with a high recruitment rate. Finally, the PI has a fully staffed research department with 10 years of experience at their disposal.

Our protocol, however, is not without limitation. Unfortunately, we are unable to blind the PI and patient; thus, the risk for confirmation bias and the Hawthorne effect cannot be eliminated, respectively. Additionally, we cannot guarantee absolute compliance with home self-directed physical therapy. To aid in patient compliance, our research department will remind study subjects via text message every morning to perform their designated home therapy protocol through our Institutional text messaging service.

\section{Trial Status}

Patient recruitment is currently open. Recruitment began in January 2019 and is expected to end by December 2020. The protocol that is being followed is version 6.0, dated $01 / 10 / 2019$.

Trial RegistrationRegistered on ClinicalTrials.gov on January 15, 2019. ClinicalTrials.gov (Identifier: NCT03804853)

URL:https://clinicaltrials.gov/ct2/show/NCT03804853? cond = reverse+total+shoulder+arthroplasty\&draw = 1\&rank $=5$

\section{List Of Abbreviations}

- American Shoulder and Elbow Society (ASES)

- Simple Shoulder Test (SST)

- Patient-Reported Outcome Information System (PROMIS)

- Reverse Total Shoulder Arthroplasty (rTSA) 
- Anatomic Total Shoulder Arthroplasty (aTSA)

- Hemi-Arthroplasty (HA)

- Immediate Active Shoulder Rehabilitation (IASR)

- Physical Therapy (PT)

- Patient Reported Outcome Measure (PROM)

- Range of Motion (ROM)

- Active Range of Motion (aROM)

- Active Assisted Range of Motion (aaROM)

- Passive Range of Motion (pROM)

- Minimal Clinical Important Difference (MCID)

- Institutional Review Board (IRB)

- National Institute of Health (NIH)

- National Library of Medicine (NLM)

- Food and Drug Administration (FDA)

- Data and Safety Monitoring Board (DSMB)

- Standard Deviation (SD)

- Progressive Resistive Exercise (PRE)

- Primary Investigator (PI)

- X-ray (XR)

\section{Declarations}

\section{Acknowledgments}

We thank the personnel (orthopaedic surgeons, research nurses, and physical therapists) from the participating departments at the TRIA Orthopaedic Center and HealthPartners Institute-Bloomington, MN 55431.

\section{Authors' contributions \{31b\}}

PD leads the coordination of the study, participated in the design of the study, and wrote this protocol manuscript. MW, AR, and TC conceived the project, led the design of the study, and contributed to the manuscript. AR provided statistical advice and assisted with drafting the relevant manuscript sections. RH and LS contributed to the manuscript, participated in study coordination, and were responsible for data acquisition. All authors provided comments on drafts of this paper and approved the final manuscript.

\section{Funding \{4\}}

HealthPartners Institute Internal Grant currently funds the study. The funding will cover indirect study costs. (i.e., coordinator time, radiologist time, administrative time, statistician time and direct costs i.e. 2 additional shoulder radiographs, 1 shoulder ultrasound and patient stipend). 


\section{Availability of data and materials $\{29\}$}

Not applicable.

\section{Ethics approval and consent to participate $\{24\}$}

Central ethical approval was confirmed from the HealthPartners Institution Review Board (ref approval no. A18259) on 20 September 2018. Funding was provided by an Internal Grant award from the HealthPartners Institute. The trial was registered at the National Institutes of Health (NIH), United States National Library of Medicine (NLM) clinical trial database (ClinicalTrials.gov, Identifier: NCT03804853) on 15 January 2019. All participants will be asked to sign an informed consent prior to initiating the study.

\section{Consent for publication $\{32\}$}

Not applicable.

\section{Competing interests $\{28\}$}

The authors declare that they have no competing interests.

\section{Author details}

1 TRIA Orthopaedic Center, Bloomington, MN, USA. ${ }^{2}$ University of Minnesota Medical School, Minneapolis, MN, USA.

\section{Bibliography}

1. Alentorn-Geli E, Clark NJ, Assenmacher AT, Samuelsen BT, Sánchez-Sotelo J, Cofield RH, et al. What are the complications, survival, and outcomes after revision to reverse shoulder arthroplasty in patients older than 80 years? Clin. Orthop. Relat. Res. 2017 Nov;475(11):2744-2751. doi:10.1007/s11999-017-5406-6

2. Bennett JP. Physical Therapy: Theory, Practices And Benefits (public Health In The 21st Century). 1st ed. New York: Nova Science Publishers, Inc.; 2011.

3. Bergen HF H. Norwegian National Advisory Unit on Arthroplasty and Hip Fractures - June 2015 Report [Internet]. The Norwegian Arthroplasty Register; 2015 [cited 2019 Nov 19]. Available from: http://nrlweb.ihelse.net/Rapporter/Report2015_english.pdf

4. Berliner JL, Regalado-Magdos A, Ma CB, Feeley BT. Biomechanics of reverse total shoulder arthroplasty. J. Shoulder Elbow Surg. 2015 Jan;24(1):150-160. doi:10.1016/j.jse.2014.08.003

5. Bigorre N, Lancigu R, Bizot P, Hubert L. Predictive factors of scapular notching in patients with reverse shoulder arthroplasty. Orthop. Traumatol. Surg. Res. 2014 Nov;100(7):711-714. doi:10.1016/j.otsr.2014.06.013

6. Blacknall J, Neumann L. Rehabilitation following Reverse Total Shoulder Replacement. Shoulder Elbow. 2011 Oct;3(4):232-240. doi:10.1111/j.1758-5740.2011.00138.x 
7. Borroff M, Green M, Gregg P, MacGregor A, Porter M, Tucker K, et al. The National Joint Registry Annual Report 2014. 2015.

8. Boudreau S, Boudreau ED, Higgins LD, Wilcox RB. Rehabilitation following reverse total shoulder arthroplasty. J. Orthop. Sports Phys. Ther. 2007 Dec;37(12):734-743. doi:10.2519/jospt.2007.2562

9. Brems JJ. Rehabilitation following total shoulder arthroplasty. Clin. Orthop. Relat. Res. 1994 Oct;(307):70-85.

10. Buchmann S, Schoch C, Grim C, Jung C, Beitzel K, Klose M, et al. Rehabilitation following reverse shoulder arthroplasty. Obere Extremität. 2019 Apr 4;1-15. doi:10.1007/s11678-019-0513-5

11. Bullock GS, Garrigues GE, Ledbetter L, Kennedy J. A systematic review of proposed rehabilitation guidelines following anatomic and reverse shoulder arthroplasty. J. Orthop. Sports Phys. Ther. 2019 May;49(5):337-346. doi:10.2519/jospt.2019.8616

12. Castricini R, Gasparini G, Di Luggo F, De Benedetto M, De Gori M, Galasso O. Health-related quality of life and functionality after reverse shoulder arthroplasty. J. Shoulder Elbow Surg. 2013 Dec;22(12):1639-1649. doi:10.1016/j.jse.2013.01.020

13. Cazeneuve JF, Cristofari DJ. Delta III reverse shoulder arthroplasty: radiological outcome for acute complex fractures of the proximal humerus in elderly patients. Orthop. Traumatol. Surg. Res. 2009 Sep;95(5):325-329. doi:10.1016/j.otsr.2009.03.018

14. Chan A-W, Tetzlaff JM, Altman DG, Laupacis A, Gøtzsche PC, Krleža-Jerić K, et al. SPIRIT 2013 statement: defining standard protocol items for clinical trials. Ann. Intern. Med. 2013 Feb 5;158(3):200-207. doi:10.7326/0003-4819-158-3-201302050-00583

15. Clark JC, Ritchie J, Song FS, Kissenberth MJ, Tolan SJ, Hart ND, et al. Complication rates, dislocation, pain, and postoperative range of motion after reverse shoulder arthroplasty in patients with and without repair of the subscapularis. J. Shoulder Elbow Surg. 2012 Jan;21(1):36-41. doi:10.1016/j.jse.2011.04.009

16. Compito CA, Self EB, Bigliani LU. Arthroplasty and acute shoulder trauma. Reasons for success and failure. Clin. Orthop. Relat. Res. 1994 Oct;(307):27-36.

17. Cuff D, Pupello D, Virani N, Levy J, Frankle M. Reverse shoulder arthroplasty for the treatment of rotator cuff deficiency. J. Bone Joint Surg. Am. 2008 Jun;90(6):1244-1251. doi:10.2106/JBJS. G.00775

18. De Biase CF, Delcogliano M, Borroni M, Castagna A. Reverse total shoulder arthroplasty: radiological and clinical result using an eccentric glenosphere. Musculoskelet. Surg. 2012 May;96 Suppl 1:S27-34. doi:10.1007/s12306-012-0193-4

19. Dedy NJ, Gouk CJ, Taylor FJ, Thomas M, Tan SLE. Sonographic assessment of the subscapularis after reverse shoulder arthroplasty: impact of tendon integrity on shoulder function. J. Shoulder Elbow Surg. 2018 Jun;27(6):1051-1056. doi:10.1016/j.jse.2017.12.008

20. Denard PJ, Lädermann A. Immediate versus delayed passive range of motion following total shoulder arthroplasty. J. Shoulder Elbow Surg. 2016 Dec;25(12):1918-1924. doi:10.1016/j.jse.2016.07.032

21. Dowdle SB, Glass N, Anthony CA, Hettrich CM. Use of PROMIS for patients undergoing primary total shoulder arthroplasty. Orthop. J. Sports Med. 2017 Sep 15;5(9):2325967117726044. doi:10.1177/2325967117726044

22. Farshad M, Gerber C. Reverse total shoulder arthroplasty-from the most to the least common complication. Int. Orthop. 2010 Dec;34(8):1075-1082. doi:10.1007/s00264-010-1125-2

23. Frankle M, Levy JC, Pupello D, Siegal S, Saleem A, Mighell M, et al. The reverse shoulder prosthesis for glenohumeral arthritis associated with severe rotator cuff deficiency. a minimum two-year follow-up study of 
sixty patients surgical technique. J. Bone Joint Surg. Am. 2006 Sep;88 Suppl 1 Pt 2:178-190. doi:10.2106/JBJS. F.00123

24. Friedman RJ, Flurin P-H, Wright TW, Zuckerman JD, Roche CP. Comparison of reverse total shoulder arthroplasty outcomes with and without subscapularis repair. J. Shoulder Elbow Surg. 2017 Apr;26(4):662668. doi:10.1016/j.jse.2016.09.027

25. Gillespie RJ, Garrigues GE, Chang ES, Namdari S, Williams GR. Surgical exposure for reverse total shoulder arthroplasty: differences in approaches and outcomes. Orthop Clin North Am. 2015 Jan;46(1):49-56. doi:10.1016/j.ocl.2014.09.015

26. Graves, PhD S, Tomkins A. Australian Orthopaedic Association National Joint Replacement Registry. Shoulder Arthroplasty Annual Report 2015. [Internet]. University of Adelaide, School of Public Health; 2015 [cited 2019 Nov 7]. Available from: https://aoanjrr.sahmri.com/documents/10180/217645/Shoulder+Arthroplasty

27. Guery J, Favard L, Sirveaux F, Oudet D, Mole D, Walch G. Reverse total shoulder arthroplasty. Survivorship analysis of eighty replacements followed for five to ten years. J. Bone Joint Surg. Am. 2006 Aug;88(8):17421747. doi:10.2106/JBJS. E.00851

28. Hsu JE, Russ SM, Somerson JS, Tang A, Warme WJ, Matsen FA. Is the Simple Shoulder Test a valid outcome instrument for shoulder arthroplasty? J. Shoulder Elbow Surg. 2017 Oct;26(10):1693-1700. doi:10.1016/j.jse.2017.03.029

29. Jung C, Tepohl L, Tholen R, Beitzel K, Buchmann S, Gottfried T, et al. Rehabilitation following rotator cuff repair: A work of the Commission Rehabilitation of the German Society of Shoulder and Elbow Surgery e. V. (DVSE) in collaboration with the German Association for Physiotherapy (ZVK) e. V., the Association Physical Therapy, Association for Physical Professions (VPT) e. V. and the Section Rehabilitation-Physical Therapy of the German Society for Orthopaedics and Trauma e. V. (DGOU). Obere Extrem. 2018 Feb 22;13(1):45-61. doi:10.1007/s11678-018-0448-2

30. Kalouche I, Sevivas N, Wahegaonker A, Sauzieres P, Katz D, Valenti P. Reverse shoulder arthroplasty: does reduced medialisation improve radiological and clinical results? Acta Orthop Belg. 2009 Apr;75(2):158-166.

31. Kempton LB, Balasubramaniam M, Ankerson E, Wiater JM. A radiographic analysis of the effects of prosthesis design on scapular notching following reverse total shoulder arthroplasty. J. Shoulder Elbow Surg. 2011 Jun;20(4):571-576. doi:10.1016/j.jse.2010.08.024

32. King JJ, Dalton SS, Gulotta LV, Wright TW, Schoch BS. How common are acromial and scapular spine fractures after reverse shoulder arthroplasty?: A systematic review. Bone Joint J. 2019;101-B(6):627-634.

doi:10.1302/0301-620X.101B6.BJJ-2018-1187.R1

33. Klein M, Juschka M, Hinkenjann B, Scherger B, Ostermann PAW. Treatment of comminuted fractures of the proximal humerus in elderly patients with the Delta III reverse shoulder prosthesis. J Orthop Trauma. 2008 Dec;22(10):698-704. doi:10.1097/BOT.0b013e31818afe40

34. Kohan EM, Chalmers PN, Salazar D, Keener JD, Yamaguchi K, Chamberlain AM. Dislocation following reverse total shoulder arthroplasty. J. Shoulder Elbow Surg. 2017 Jul;26(7):1238-1245. doi:10.1016/j.jse.2016.12.073

35. Kwaees TA, Charalambous CP. Reverse shoulder arthroplasty-minimum age for surgery, postoperative rehabilitation and long term restrictions. A delphi consensus study. Ortop Traumatol Rehabil. 2014 Aug;16(4):435-439. doi:10.5604/15093492.1119621

36. Leung B, Horodyski M, Struk AM, Wright TW. Functional outcome of hemiarthroplasty compared with reverse total shoulder arthroplasty in the treatment of rotator cuff tear arthropathy. J. Shoulder Elbow Surg. 2012 
Mar;21(3):319-323. doi:10.1016/j.jse.2011.05.023

37. Lévigne C, Garret J, Boileau P, Alami G, Favard L, Walch G. Scapular notching in reverse shoulder arthroplasty: is it important to avoid it and how? Clin. Orthop. Relat. Res. 2011 Sep;469(9):2512-2520. doi:10.1007/s11999-010-1695-8

38. Melis B, DeFranco M, Lädermann A, Molé D, Favard L, Nérot C, et al. An evaluation of the radiological changes around the Grammont reverse geometry shoulder arthroplasty after eight to 12 years. J. Bone Joint Surg. Br. 2011 Sep;93(9):1240-1246. doi:10.1302/0301-620X.93B9.25926

39. Mizuno N, Denard PJ, Raiss P, Walch G. The clinical and radiographical results of reverse total shoulder arthroplasty with eccentric glenosphere. Int. Orthop. 2012 Aug;36(8):1647-1653. doi:10.1007/s00264-012$1539-0$

40. Palsis JA, Simpson KN, Matthews JH, Traven S, Eichinger JK, Friedman RJ. Current trends in the use of shoulder arthroplasty in the united states. Orthopedics. 2018 May 1;41(3):e416-e423. doi:10.3928/01477447-20180409-05

41. Reitman RD, Kerzhner E. Reverse shoulder arthoplasty as treatment for comminuted proximal humeral fractures in elderly patients. Am J Orthop. 2011 Sep;40(9):458-461.

42. Rhee YG, Cho NS, Moon SC. Effects of humeral component retroversion on functional outcomes in reverse total shoulder arthroplasty for cuff tear arthropathy. J. Shoulder Elbow Surg. 2015 Oct;24(10):1574-1581. doi:10.1016/j.jse.2015.03.026

43. Richards RR, An KN, Bigliani LU, Friedman RJ, Gartsman GM, Gristina AG, et al. A standardized method for the assessment of shoulder function. J. Shoulder Elbow Surg. 1994 Nov;3(6):347-352. doi:10.1016/S10582746(09)80019-0

44. Roberts CC, Ekelund AL, Renfree KJ, Liu PT, Chew FS. Radiologic assessment of reverse shoulder arthroplasty. Radiographics. 2007 Feb;27(1):223-235. doi:10.1148/rg.271065076

45. Romano AM, Oliva F, Nastrucci G, Casillo P, Di Giunta A, Susanna M, et al. Reverse shoulder arthroplasty patient personalized rehabilitation protocol. Preliminary results according to prognostic groups. Muscles Ligaments Tendons J. 2017 Jun;7(2):263-270. doi:10.11138/mltj/2017.7.2.263

46. Rosenberg/ThoughtCo M. Most Populous Metropolitan Areas in the United States: The 30 Largest Metropolitan Areas in the United States [Internet]. ThoughtCo. 2019 Jun 17 [cited 2019 Nov 26];Available from: https://www.thoughtco.com/largest-metropolitan-areas-1435135

47. Ross M, Hope B, Stokes A, Peters SE, McLeod I, Duke PFR. Reverse shoulder arthroplasty for the treatment of three-part and four-part proximal humeral fractures in the elderly. J. Shoulder Elbow Surg. 2015 Feb;24(2):215222. doi:10.1016/j.jse.2014.05.022

48. Routman HD. Indications, technique, and pitfalls of reverse total shoulder arthroplasty for proximal humerus fractures. Bull Hosp Jt Dis (2013). 2013;71 Suppl 2:64-67.

49. Roy J-S, Macdermid JC, Faber KJ, Drosdowech DS, Athwal GS. The simple shoulder test is responsive in assessing change following shoulder arthroplasty. J. Orthop. Sports Phys. Ther. 2010 Jul;40(7):413-421. doi:10.2519/jospt.2010.3209

50. Simovitch R, Flurin P-H, Wright T, Zuckerman JD, Roche CP. Quantifying success after total shoulder arthroplasty: the minimal clinically important difference. J. Shoulder Elbow Surg. 2018 Feb;27(2):298-305. doi:10.1016/j.jse.2017.09.013 
51. Sirveaux F, Favard L, Oudet D, Huquet D, Walch G, Molé D. Grammont inverted total shoulder arthroplasty in the treatment of glenohumeral osteoarthritis with massive rupture of the cuff. Results of a multicentre study of 80 shoulders. J. Bone Joint Surg. Br. 2004 Apr;86(3):388-395. doi:10.1302/0301-620x.86b3.14024

52. Sofka CM, Adler RS. Original report. Sonographic evaluation of shoulder arthroplasty. AJR Am. J. Roentgenol. 2003 Apr;180(4):1117-1120. doi:10.2214/ajr.180.4.1801117

53. Sperling JW, Cofield RH, O'Driscoll SW, Torchia ME, Rowland CM. Radiographic assessment of ingrowth total shoulder arthroplasty. J. Shoulder Elbow Surg. 2000 Dec;9(6):507-513. doi:10.1067/mse.2000.109384

54. Tashjian RZ, Hung M, Keener JD, Bowen RC, McAllister J, Chen W, et al. Determining the minimal clinically important difference for the American Shoulder and Elbow Surgeons score, Simple Shoulder Test, and visual analog scale (VAS) measuring pain after shoulder arthroplasty. J. Shoulder Elbow Surg. 2017 Jan;26(1):144148. doi:10.1016/j.jse.2016.06.007

55. Walch G, Mottier F, Wall B, Boileau P, Molé D, Favard L. Acromial insufficiency in reverse shoulder arthroplasties. J. Shoulder Elbow Surg. 2009 Jun;18(3):495-502. doi:10.1016/j.jse.2008.12.002

56. Walker M, Willis MP, Brooks JP, Pupello D, Mulieri PJ, Frankle MA. The use of the reverse shoulder arthroplasty for treatment of failed total shoulder arthroplasty. J. Shoulder Elbow Surg. 2012 Apr;21(4):514-522. doi:10.1016/j.jse.2011.03.006

57. Wall B, Nové-Josserand L, O'Connor DP, Edwards TB, Walch G. Reverse total shoulder arthroplasty: a review of results according to etiology. J. Bone Joint Surg. Am. 2007 Jul;89(7):1476-1485. doi:10.2106/JBJS. F.00666

58. Werner CML, Steinmann PA, Gilbart M, Gerber C. Treatment of painful pseudoparesis due to irreparable rotator cuff dysfunction with the Delta III reverse-ball-and-socket total shoulder prosthesis. J. Bone Joint Surg. Am. 2005 Jul;87(7):1476-1486. doi:10.2106/JBJS. D.02342

59. Wilcox RB, Arslanian LE, Millett P. Rehabilitation following total shoulder arthroplasty. J. Orthop. Sports Phys. Ther. 2005 Dec;35(12):821-836. doi:10.2519/jospt.2005.35.12.821

60. Wolff AL, Rosenzweig L. Anatomical and biomechanical framework for shoulder arthroplasty rehabilitation. J. Hand Ther. 2017;30(2):167-174. doi:10.1016/j.jht.2017.05.009

61. Wylie JD, Beckmann JT, Granger E, Tashjian RZ. Functional outcomes assessment in shoulder surgery. World J Orthop. 2014 Nov 18;5(5):623-633. doi:10.5312/wjo.v5.i5.623

\section{Supplementary Files Legend}

Additional File 1: IASR post-operative protocol.

Additional File 2: Standard care post-operative protocol.

Additional File 3: Post-operative exercise handout.

\section{Figures}




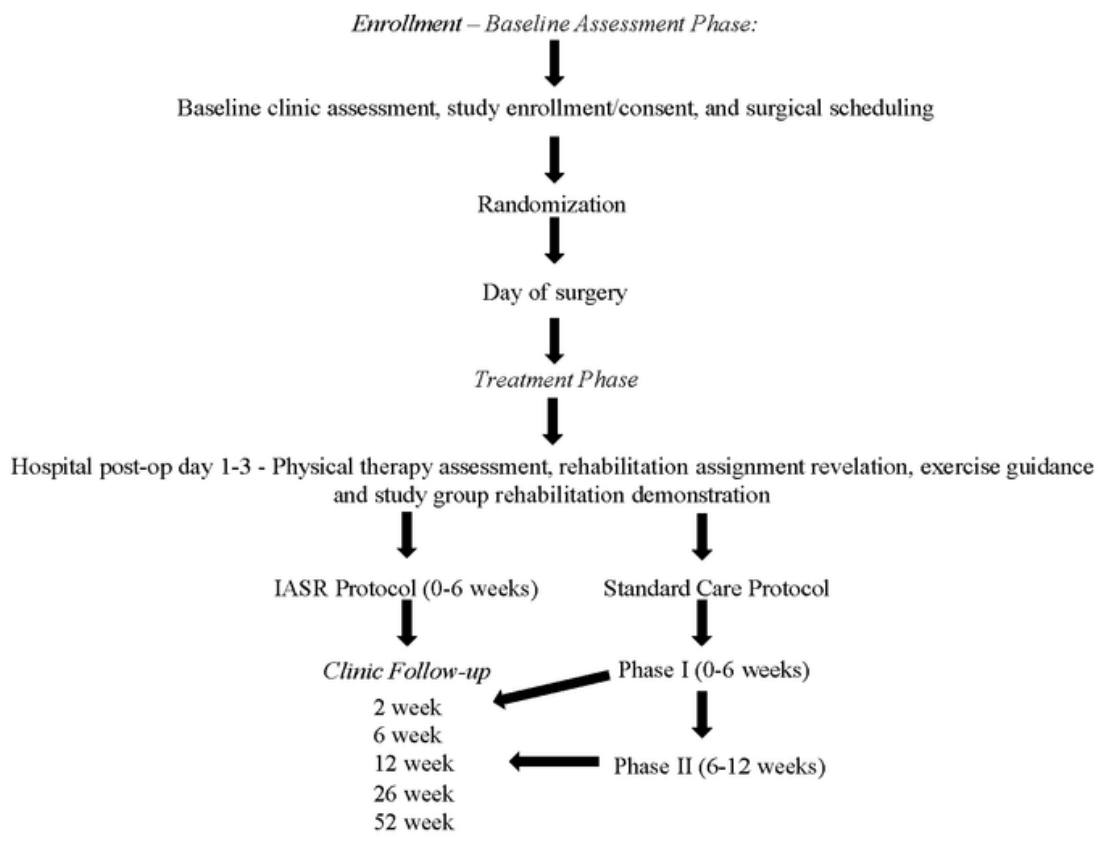

Figure 1

IASR vs. standard therapy trial study patient flowchart.

\section{Supplementary Files}

This is a list of supplementary files associated with this preprint. Click to download.

- PostoperativeExerciseHandoutAdditionalFile3.pdf

- StandardCareProtocolAdditionalFile2.pdf

- IASRProtocolAdditionalFile1.pdf 